\title{
Rethinking the significance of the superficial pus swab in the emergency setting
}

Christoffel J Opperman*

Division of Medical Microbiology, National Health Laboratory Service, University of Cape Town and Groote Schuur Hospital, Cape Town, South Africa

*Email: Stefanopperman1@gmail.com

Check for updates

Received 1105 2018; Accepted 26072018

A superficial pus swab is a valuable tool if taken correctly. Poorly taken specimens tend to be heavily contaminated and do not financially justify susceptibility testing of numerous different organisms. Most superficial pus swabs taken in the emergency centre have more than three microorganisms cultured and no predominant growth of pathogenic organisms. The microbiology laboratory will report mixed growth with questionable significance and request a repeat specimen if clinically indicated. Mixed cultures are difficult to interpret by the pathologist and of little value to the clinician. It is important to stress that superficial pus swabs are inferior to tissue biopsies and aspirated pus collections as anaerobic and fastidious organisms tend to die in the aerobic environment of the pus swabs. ${ }^{1}$ Therefore, the significance of the superficial pus swab in the emergency care setting is brought into question.

An audit was done in the Department of Pathology, Microbiology Division, National Health Laboratory Service, Groote Schuur Hospital on superficial pus swabs taken from emergency units during 2016 and 2017. From the 197 superficial pus swabs taken in 2016 only $17.2 \%$ were relevant, the rest were sent out as mixed growth or skin contamination. This value increased to $18.5 \%$ during 2017 from a total of 183 superficial pus swabs collected. Staphylococcus aureus and Streptococcus pyogenes were the most prominent pathogenic microorganisms cultured in 2016 and 2017 from soft-tissue infections (Figure 1). This may justify covering empirically for these organisms if clinically indicated and if the infection is related to soft tissue. Most swabs taken in the casualty department are from the surface of wounds and represent contamination or colonisation. Skin flora or mixed growth was present in $82.8 \%$ of emergency centre specimens in 2016 and $81.5 \%$ in 2017.

Superficial swabs are user friendly and readily available in the emergency department. It should be remembered that every positive wound swab culture does not represent an active infection and must be correlated with the clinical picture. Wound debridement or proper cleaning of the area with sterile saline remains important before sample collection to reduce the yield of poly-microbial overgrowth. ${ }^{1}$ The $Z$ technique (moving the pus swab in a zig-zag fashion over the wound without touching the edges) is no longer recommended due to its low sensitivity $(63 \%)$ and specificity (53\%). ${ }^{2}$ This has been replaced by Levine's technique (rotating the swab for 5 seconds over a 1 $\mathrm{cm}^{2}$ area with sufficient pressure to extract inner wound fluid) with a sensitivity of $91 \%$ and specificity of $57 \%$, respectively. 2,3 The site of specimen collection must be indicated on the laboratory request form to facilitate the appropriate selection of media

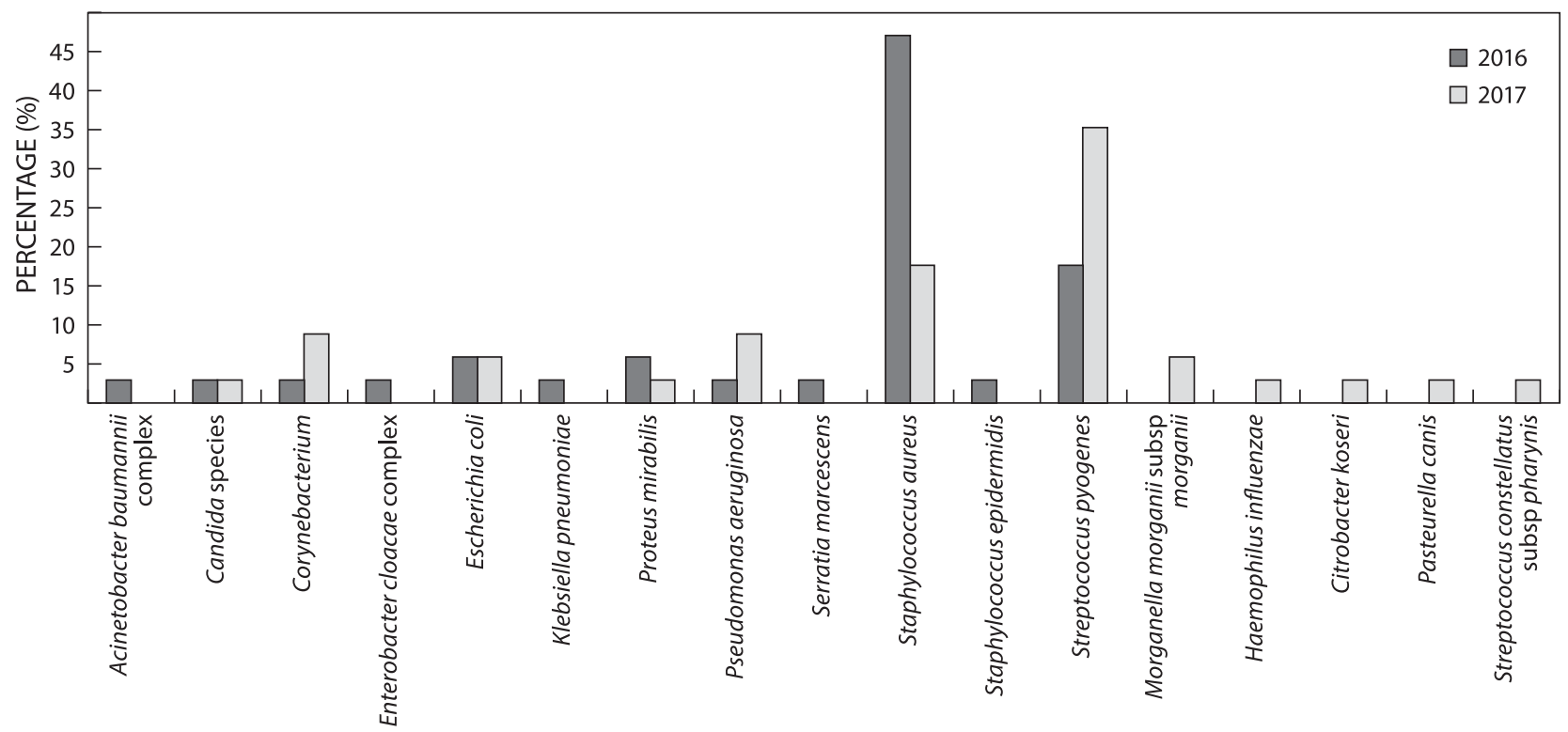

Figure 1: Percentage of pathogens isolated from superficial pus swabs, not reported as mixed growth or skin contamination, taken in emergency centres and processed in the National Health Laboratory Service, Department of Pathology, Division of Microbiology, Groote Schuur Hospital during 2016 and 2017. 
and growth conditions. Superficial pus swabs ought to be taken aseptically before the commencement of antibiotic therapy.

It is concluded that the superficial pus swab still plays an important role in pathogen identification in the emergency setting if done correctly with a diligently completed laboratory request form.

Disclosure statement - No potential conflict of interest was reported by the author.

\section{References}

1. Orth H. Interpretation of microbiological reports. Contin Med Educ. 2008;26(11):545-547.

2. Cross $\mathrm{HH}$. Obtaining a wound swab culture specimen. Nursing. 2014:44(7):68-69. doi:10.1097/01.NURSE.0000446645.33489.2e.

3. Huang $Y$, Cao Y, Zou M, et al. A comparison of tissue versus swab culturing of infected diabetic foot wounds. Int J Endocrinol. 2016;2016:8198714. doi:10.1155/2016/8198714.

Received: 11-05-2018 Accepted: 26-07-2018 